\title{
Musique et conflits armés après 1945
}

Music and Armed Conflicts after 1945

\section{(2) OpenEdition}

\section{Journals}

Édition électronique

URL : http://journals.openedition.org/transposition/1057

DOI : 10.4000/transposition. 1057

ISSN : 2110-6134

Éditeur

CRAL - Centre de recherche sur les arts et le langage

Référence électronique

" Musique et conflits armés après 1945 », Transposition [En ligne], 4 | 2014, mis en ligne le 15 juillet 2014, consulté le 16 février 2020. URL : http://journals.openedition.org/transposition/1057 ; DOI : 10.4000/transposition. 1057

Ce document a été généré automatiquement le 16 février 2020.

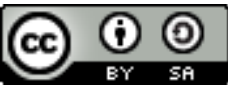

La revue Transposition est mise à disposition selon les termes de la Licence Creative Commons Attribution - Partage dans les Mêmes Conditions 4.0 International. 


\title{
Musique et conflits armés après 1945
}

\author{
Music and Armed Conflicts after 1945
}

1 Dans son ouvrage De la guerre, paru pour la première fois en 1832, Carl von Clausewitz affirme que la guerre se situe à l'intérieur des rapports politiques: elle serait un véritable «instrument politique, une continuation des rapports politiques, la réalisation des rapports politiques par d'autres moyens ${ }^{1} »$. Ainsi, l'un des apports les plus importants de la pensée de Clausewitz à la compréhension de la guerre, résultant de ce postulat, serait que celle-ci ne constitue pas la rupture d'un processus, un moment liminaire en dehors des rapports sociaux et politiques, mais leur continuation - « la politique existe avant la guerre, elle se poursuit à travers la guerre dans la décision d'engagement des forces armées et continue après la guerre; à aucun moment son cours n'est interrompu ${ }^{2}$.

2 Inversement, la guerre agit sur le politique ${ }^{3} \mathrm{du}$ fait qu'elle a la capacité de reconfigurer durablement les rapports sociaux, les cadres sensibles, les codes symboliques et les dispositifs mémoriels des sociétés concernées. Elle implique donc une mobilisation de ressources symboliques, de discours et de pratiques de la part des acteurs qui va bien au-delà de l'engagement armé et des violences qui en découlent. C'est ainsi que la réflexion sur les rapports entre les conflits armés, d'une part, et les œuvres et les pratiques musicales (en tant que ressources et objets symboliques) d'autre part, peut être d'une grande utilité pour penser aussi bien les processus mis en œuvre dans les dynamiques de la violence armée que les processus de création, d'appropriation et de mobilisation propres à l'objet musical.

3 Les recherches historiques et musicologiques sur le rôle des œuvres et des pratiques musicales au sein des conflits armés - ainsi que celles sur l'impact des conflits sur les œuvres et les pratiques musicales - ont connu un essor significatif durant les trois dernières décennies ${ }^{4}$, notamment en ce qui concerne les deux guerres mondiales du $\mathrm{XX}^{\mathrm{e}}$ siècle $^{5}$. De même, les recherches sur les liens entre musique et violence ${ }^{6}$ ou musique et contestation ${ }^{7}$, ainsi que sur les rapports entre la musique et les régimes autoritaires et totalitaires ${ }^{8}$ ont eu une influence - directe ou indirecte - sur le développement de cet objet d'étude. Enfin, les recherches sur les rapports entre la 
musique et les conflits armés contemporains ont trouvé une place significative au sein des conflict transformation studies ${ }^{9}$, lesquelles ont participé à la consolidation d'une approche interdisciplinaire où l'objet musical occupe une place centrale. Le dossier du quatrième numéro de Transposition. Musique et sciences sociales participe du développement de cette réflexion sur les rapports entre les œuvres, les pratiques musicales et les conflits armés d'après 1945. Ce choix chronologique se justifie par la relative absence de recherches en musicologie sur les conflits armés contemporains, en regard notamment de la richesse des travaux traitant par exemple des deux guerres mondiales. Il a semblé nécessaire au comité de rédaction de Transposition d'ouvrir ici un espace de réflexion qui aborderait le rôle des œuvres et des pratiques musicales dans divers contextes géopolitiques, au sein de différentes zones géographiques dans lesquelles la violence armée s'est établie, mais qui traiterait aussi de l'impact des conflits contemporains sur la création musicale elle-même ${ }^{10}$.

De manière générale, les articles qui constituent ce numéro peuvent être regroupés en suivant trois axes différents mais non exclusifs : ceux qui analysent le déclenchement des processus d'identification des différents acteurs engagés dans les conflits armés par le biais de la création ou de la mobilisation d'œuvres et de pratiques musicales; ceux qui étudient les utilisations et les appropriations des œuvres musicales par des acteurs engagés dans la violence armée ; ceux, enfin, qui analysent la fonction sociale qu'ont les œuvres et les pratiques musicales de sublimer la souffrance provoquée par ces conflits. Ces textes mettent en exergue la participation des œuvres et des pratiques musicales à la ritualisation des actions et violences liées aux conflits armés, ainsi qu'aux modalités mémorielles qui en découlent: l'entraînement des combattants, l'engagement des musiciens vis-à-vis de la guerre, les pratiques de torture, les processus de consolidation des imaginaires sociaux ou encore la sublimation de la tristesse associée au deuil.

5 L'article de Panagiota Anagnostou analyse ainsi les débats provoqués par le rebetiko dans la presse de gauche dans le contexte de la guerre civile grecque. L'auteure étudie comment la catégorie de "peuple» se reconfigure dans les débats des intellectuels grecs de l'après-guerre à propos du rebetiko et de quelle façon la recherche d'une musique " authentiquement populaire » vient remplacer « la question de la "musique nationale", qui a intensément préoccupé les intellectuels de toute orientation depuis la fin du XIX siècle ». S'appuyant sur une vaste documentation, Anagnostou examine le rôle de la musique dans les processus d'institutionnalisation de la mémoire sociale liée au conflit armé grec. Elle montre de quelle façon « la construction de la catégorie semiimaginaire du "vrai" rebetiko suscite un intérêt pour le passé, un intérêt conforme à l'époque: plus on s'éloigne de la défaite lors de la guerre civile, plus le besoin de comprendre et, jusqu'à un certain point, de remodeler le passé récent apparaît ».

6 La capacité de la musique à enclencher des processus d'identification est aussi analysée par Mauricio Gómez Gálvez à propos de l'engagement des musiciens chiliens contre la guerre du Vietnam. L'analyse de l'auteur part de l'hypothèse selon laquelle, dans le contexte du Chili de Salvador Allende, « la lutte contre la guerre du Vietnam, en tant que facteur majeur de mobilisation et de cohésion sociale, joue un rôle important dans l'éclosion de formes d'art politiquement engagé, y compris la musique, consolidant - et préfigurant parfois - les manifestations les plus caractéristiques de la période de l'Unité Populaire». L'auteur étudie les modalités et la portée de l'opposition des musiciens chiliens contre la guerre du Vietnam, notamment les représentants de la 
Nouvelle chanson chilienne, montrant la pluralité des genres et des styles musicaux mobilisés.

7 Pour sa part, l'article de Luis Velasco-Pufleau explore les rapports entre idéologie, technologie et conflits armés dans l'œuvre Für Paul Dessau de Luigi Nono. Pour l'auteur, cette œuvre "répond à la problématique concrète de la politisation de la musique durant la Guerre froide: l'utilisation stratégique de la création musicale et de la technologie au service d'un idéal politique». Ainsi, s'appuyant sur les notions d'idéologie, d'intellectuel et d'hégémonie, Velasco-Pufleau analyse de quelle façon Nono identifie et mobilise les luttes de libération du tiers-monde dans son œuvre et étudie les enjeux esthétiques et politiques de l'engagement communiste du compositeur italien. Il montre enfin comment, "grâce aux moyens offerts par l'électronique, Für Paul Dessau "transpose" et relie symboliquement les voix d'un archipel de luttes politiques et de conflits armés du XX $\mathrm{XX}^{\mathrm{e}}$ siècle ».

8 Cornelia Nuxoll examine de son côté la question du transfert des pratiques musicales en contexte de violence armée, via l'appropriation et l'usage des chants de commandement et de motivation par les combattants du Revolutionary United Front (RUF) durant la guerre civile en Sierra Leone. À partir d'une approche socioanthropologique, l'auteure souligne l'influence, dans ce conflit, du répertoire et des pratiques musicales initialement développés lors de la guerre civile au Libéria, en étudiant l'adaptation contrefactuelle des paroles et mélodies des chants mais aussi leur efficacité émotionnelle dans l'entraînement des combattants du RUF et dans l'enclenchement de la violence armée. La musique fait partie des rituels quotidiens qui permettent aux combattants d'affronter et d'appréhender collectivement l'expérience de la guerre.

$9 \quad$ Les usages des œuvres et des pratiques musicales pour encadrer ou pour canaliser la violence armée sont multiples et ont une histoire spécifique, ainsi que le montre le texte de Morag Josephine Grant concernant l'utilisation de la musique dans des pratiques de torture. L'auteure analyse, dans cet article, cinq voies différentes - mais interconnectées - de ces usages, certaines très anciennes, comme la tradition militaire, ou d'autres plus récentes, comme l'utilisation de la musique dans les pratiques de privation sensorielle développées durant la guerre froide et mises en œuvre actuellement, notamment par l'armée des États-Unis dans le cadre de leur «guerre contre la terreur ». Grant analyse cette évolution dans une perspective historique, soulignant les rapports de domination et les conceptions philosophiques de l'être humain qui sous-tendent cette utilisation de la musique, ainsi que la dimension politique de ces pratiques.

10 Cette utilisation récente de la musique comme instrument de torture, notamment par l'armée des États-Unis, a provoqué d'importants débats outre-Atlantique ${ }^{11}$. Afin de faire part aux lecteurs francophones des problématiques éthiques, artistiques, politiques et sociales soulevées par ces pratiques, le comité de rédaction de Transposition a décidé de traduire et de publier en français un article important de Suzanne G. Cusick sur le sujet $^{12}$. Dans ce texte, l'auteure livre des témoignages d'anciens prisonniers et d'anciens militaires ayant subi ou pratiqué ces méthodes en Irak, en Afghanistan, au Pakistan et à Cuba. Elle y montre le caractère systématique et réfléchi de ces pratiques de torture, rendant compte des spécificités et des conséquences de l'implication de la musique dans ces actes. Les recherches de Cusick ont eu un impact considérable sur la connaissance publique de ces méthodes, ainsi que sur la condamnation de l'utilisation 
de la musique dans tout acte de torture de la part de la communauté académique et artistique aux États-Unis ${ }^{13}$. Suite à la demande du comité de rédaction de Transposition, Suzanne G. Cusick a écrit un texte sous forme d'épilogue pour accompagner la publication de cette traduction. Elle y fait un état des lieux des principales recherches menées depuis la publication de son article en 2008.

Ce dossier thématique est accompagné d'un article de Jillian Rogers, qui porte sur les liens entre la création musicale de Maurice Ravel, la pratique du piano de Marguerite Long et le processus de deuil en France dans le contexte de la Première Guerre mondiale. Dans ce texte, l'auteure analyse comment la pratique du piano était considérée, dans la France de la Grande Guerre, comme salutaire pour affronter la tristesse provoquée par l'expérience de la guerre. Elle montre ensuite de quelle façon, « en composant Le Tombeau de Couperin dans le style du jeu perlé que son amie Marguerite Long préférait, Ravel lui a donné l'occasion de faire [le] deuil [de son époux mort au combat en 1914] à travers un jeu pianistique répétitif, rythmique et exigeant sur le plan kinesthésique, susceptible, pensait-on, d'engendrer une transformation émotionnelle ». Cet article met ainsi en perspective cette dimension émotionnelle des pratiques musicales dans leur interaction avec l'expérience collective de la guerre et la douloureuse intimité du deuil.

Le numéro est complété par deux entretiens avec des chercheurs français - Florence Gétreau et Didier Francfort - qui ont travaillé, ou qui travaillent, sur les liens entre la musique et les conflits armés. Dans le premier entretien, Didier Francfort partage avec nous l'importance qu'a eue la musique dans son parcours d'historien, notamment dans la consolidation d'une approche comparée des faits historiques et des pratiques culturelles, et ouvre un dialogue sur la mobilisation par des acteurs divers des œuvres et des pratiques musicales au sein des conflits armés. Dans le second entretien, Florence Gétreau retrace son parcours de chercheuse et de conservatrice de musée et présente certains de ses projets actuels, notamment l'exposition Entendre la guerre: sons, musiques et silence en 14-18, organisée par l'Historial de Péronne à l'occasion du centenaire de la Première Guerre mondiale.

13 À partir de ce numéro, Transposition. Musique et sciences sociales sera publiée sur la plateforme de revues en sciences humaines et sociales revues.org. Cette nouvelle étape dans la vie de la revue réaffirme l'engagement du comité de rédaction en faveur de la diffusion en libre accès des recherches originales en musicologie et en sciences sociales portant sur les différentes facettes de l'objet musical.

\section{NOTES}

1. CLAUSEWITZ, Carl von, De la guerre, édition abrégée et présentée par Gérard de Chailand, traduction de l'allemand par Laurent Murawiec, Paris, Perrin, 2006, p. 56.

2. DESPORTES, Vincent, Comprendre la guerre, Paris, Economica, 2001, p. 17. 
3. Entendu ici dans le sens que lui donne Jacques Rancière : «l'activité qui reconfigure les cadres sensibles au sein desquels se définissent des objets communs" (RANCIÈrE, Jacques, "Les paradoxes de l'art politique", in Le Spectateur émancipé, Paris, La Fabrique, 2008, p. 66).

4. À titre indicatif, voir le récent ouvrage dont le compte-rendu est publié dans ce numéro, GRANT, Morag J. et STONE-DAVIS, Férdia J. (éd.), The Soundtrack of Conflict: The Role of Music in Radio Broadcasting in Wartime and in Conflict Situations, Hildesheim, Georg Olms Verlag, 2013.

5. Voir par exemple AUDoIN-ROUZEAU, Stéphane, BUCH, Esteban, CHIMÈNES, Myriam et DURosoIr, Georgie (dir.), La Grande Guerre des musiciens, Lyon, Symétrie, coll. «Perpetuum mobile », 2009 ; DOÉ DE MAINDREVILLE, Florence, ETCHARRY, Stéphan (dir.), La Grande Guerre en musique. Vie et création musicales en France pendant la Première Guerre mondiale, Bruxelles, P.I.E. Peter Lang, coll. « Études de Musicologie/Musicological Studies », 2014 ; FAUSER, Annegret, Sounds of War. Music in the United States during World War II, New York, Oxford, Oxford University Press, 2013.

6. Par exemple, voir à ce sujet le numéro 10 de Trans. Revista intercultural de música (en ligne, http://www.sibetrans.com/trans/publicacion/5/trans-10-2006) ainsi que FAST, Susan et PEGLEY, Kip (éd.), Music, Politics and Violence, Middletown, Westleyan University Press, 2012.

7. Voir par exemple KUTSCHKE, Beate et NORTON, Barley (éd.), Music and Protest in 1968, Cambridge, New York, Cambridge University Press, 2013.

8. Sur ce sujet, voir plusieurs articles de la revue en ligne Music and Politics (http:// quod.lib.umich.edu/m/mp) ou l'ouvrage suivant : ILLIANo, Roberto et SALA, Massimiliano (éd.), Music and Dictatorship in Europe and Latin America, Turnhout, Brepols, 2009.

9. Voir à ce sujet BERGH, Arild et SLOBODA, John, «Music and Art in Conflict Transformation: A Review ", in Music and Arts in Actions, Vol. 2, n 2, 2010 (en ligne, http://musicandartsinaction.net/index.php/maia/article/view/ conflicttransformation).

10. Ce numéro est resté cependant ouvert aux recherches qui nous ont paru originales sur les conflits armés d'avant 1945, comme le montre l'article de Jillian Rogers.

11. Pour un aperçu de ces débats, voir deux articles traduits en français dans Courrier international : $\quad$ http://www.courrierinternational.com/article/2006/02/23/de-lamusique-comme-instrument-de-torture ; http://www.courrierinternational.com/ article/2009/10/26/des-artistes-s-elevent-contre-la-torture-musicale.

12. Pour une discussion en français sur cette problématique, voir aussi VOLCLER, Juliette, Le Son comme arme. Les usages policiers et militaires du son, Paris, La Découverte, 2011 ; SZENDY, Peter, « Musique et torture (1). Les stigmates du son ", in Po\&sie, $\mathrm{n}^{\circ} 134$, Belin, 2010, p. 85-92 ; SZENDY, Peter, "Musique et torture (2). Cloués au sens », in Po\&sie, nº 135, Belin, 2011, p. 109-115.

13. Par exemple, voir la déclaration de la Society for American Music pour condamner et protester contre ces usages (http://american-music.org/organization/ tick_resolution.php). 